\title{
Bjomédica
}

Articulo Original

\section{Adherencia al tratamiento terapéutico en pacientes con enfermedad de Chagas del Estado de Morelos.}

Ricardo Arce-Vega1, Angélica Ángeles-Llerenas2, Alejandro Villegas-Trejo3, Celso Ramos*1.

1Centro de Investigaciones sobre Enfermedades Infecciosas, Instituto Nacional de Salud Pública (INSP), Av. Universidad 655, Col. Santa María Ahuacatitlán, 62100 Cuernavaca, Morelos, 2Centro de Investigación en Salud Poblacional, INSP, 3 Asesoría y Capacitación en Salud Pública y Enfermedades Transmitidas por Vectores, Cuernavaca, Mor.

\section{Autor para correspondencia}

Nombre: Dr. Celso Ramos

Institución: Instituto Nacional de Salud Pública

Dirección: Av. Universidad No. 655

Colonia Santa María Ahuacatitlán

62100 Cuernavaca, Morelos

Tel. 7773293000 ext. 2331

E-mail: cramos@insp.mx

Recibido: el 26 de agosto de 2016 Aceptado para publicación: el 06 de enero de 2017

Copyright () 2016 por autores (s) y Revista Biomédica.

Este trabajo esta licenciado bajo las atribuciones de la Creative Commons (CC BY).

http://creativecommons.org/licenses/by/4.0/

(c) (9) Open Access 
Arce-Vega et al.

\section{Adherencia al tratamiento en pacientes con Chagas}

\section{Resumen}

Introducción. La enfermedad de Chagas es endémica en el estado de Morelos. El medicamento disponible es el benznidazol, sin embargo generalmente la adherencia al tratamiento no es vigilado por el personal médico para verificar su cumplimiento.

Objetivo. Evaluar la adherencia al tratamiento terapéutico y los factores de riesgo asociados con su cumplimiento y el registro de efectos adversos en pacientes con enfermedad de Chagas del estado de Morelos.

Material y Métodos. Estudio de seguimiento en 20 pacientes asintomáticos. La adherencia se evaluó mediante la aplicación del cuestionario estructurado de Morisky-Green, se aplicó un cuestionario para registrar los factores de riesgo relacionados con el apego al tratamiento, se hicieron visitas domiciliarias y se hicieron entrevistas personalizadas a 8 casos para obtener información sobre factores positivos y negativos asociados al tratamiento.

Resultados. El 65\% de los pacientes fueron adherentes al tratamiento y 35\% fueron no adherentes debido a conductas de incumplimiento. Siete casos (35\%) presentaron algún tipo de reacción adversa al medicamento que no ameritó la suspensión del tratamiento, excepto dos casos que tuvieron reacciones severas. El análisis estadístico de las variables en los casos adherentes y no adherentes no mostró asociación estadística entre la adherencia y las variables de nivel socioeconómico, escolaridad, acceso al servico médico, estado civil, edad y sexo. Conclusiones. La evaluacion de la adherencia en pacientes con diagnóstico confirmado de enfermedad de Chagas del estado de Morelos, aportó información para dar seguimiento al cumplimiento del tratamiento y registrar los efectos adversos.

Palabras Clave: enfermedad de Chagas, tratamiento, adherencia.

\section{Abstract}

\section{Adherence to treatment of Chagas disease}

Introduction. Chagas disease is endemic in the state of Morelos. The available medication is benznidazole, however adherence to treatment is generally not monitored by medical personnel to verify the accomplishment. 
Objective. To evaluate the adherence to therapeutic treatment and the risk factors associated with its compliance and the registry of adverse effects in patients with Chagas disease in the state of Morelos.

Material and Methods. Follow-up study in 20 chronic asymptomatic patients. Adherence was assessed using the Morisky-Green structured test, a questionnaire was applied to record the risk factors related to adherence to treatment, home visits were made to the cases, and personalized interviews were conducted in 8 cases to obtain information on positive and negative factors associated with treatment.

Results. Sixty five percent of patients were adherent to treatment and $35 \%$ were nonadherent due to non-compliance behaviors. Seven cases (35\%) had some type of adverse reaction to the drug that did not warrant treatment discontinuation, except for two cases that had severe reactions. Statistical analysis of the variables in adherent and non-adherent cases showed no statistical association between adherence and variables of socioeconomic level, school level, access to medical service, marital status, age and sex.

Conclusions. Adherence assessment in patients with a confirmed diagnosis of Chagas' disease in the state of Morelos provided information to follow up on treatment compliance and record of adverse effects.

Keywords: : Chagas disease, treatment, adherence. 
Arce-Vega et al.

\section{Introducción}

La enfermedad de Chagas (EC) es una patología endémica de América Latina donde afecta alrededor de 7 millones de personas, más de 100 millones están en riesgo de adquirirla y mueren más de 13,000 personas al año por esta causa (1). Recientemente las migraciones humanas de zonas endémicas han facilitado su dispersión en Estados Unidos de América (EUA), Europa y Asia (2-4). En los EUA se estima que alrededor de 300,000 migrantes latinoamericanos están infectados y más de 45,000 padecen cardiomiopatía, sin embargo la gran mayoría no son diagnosticados y tratados (5).

En México, la enfermedad de Chagas es un problema de salud pública por su amplia distribución e impacto en la población; se estima que en el país existen alrededor de 1 millón de casos infectados, cerca de medio millón de casos crónicos y aproximadamente 65,000 casos nuevos por año $(6,7)$. En 2016, la Dirección General de Epidemiología de la Secretaría de Salud, reportó 805 casos de enfermedad de Chagas (EC) distribuidos principalmente en Chiapas, Hidalgo, Jalisco, Estado de México, Morelos, Oaxaca, Tamaulipas, Veracruz y Yucatán (8). En el período de 2007-2011, se reportaron en el Estado de Morelos 263 casos de EC, de los cuales más de la mitad recibieron tratamiento $(6,7)$, pero se desconoce su situación actual ya que generalmente no se da seguimiento a los casos.

Clínicamente la enfermedad de Chagas se caracteriza por una fase aguda, generalmente asociada con parasitemia y sintomatología leve, seguida por una fase asintomática indeterminada que puede durar mucho tiempo. Sin embargo alrededor del $30 \%$ de los casos indeterminados sin tratamiento pueden progresar a la fase crónica, que puede asociarse con afectación cardíaca (cardiopatía chagásica crónica) y de otras megavísceras (p.e. megacolon y megaesófago) (9).

Actualmente existen dos medicamentos (nifurtimox y benznidazol) para el tratamiento de la enfermedad de Chagas. Ambos medicamentos son más efectivos en los niños y jóvenes y en las etapas tempranas de la infección; la tasa de curación en los recién nacidos infectados congénitamente es cerca del 100\% (10). En los países endémicos y no endémicos se usan indistintamente ambos medicamentos cuyos efectos terapéuticos son variables, sin embargo, a nivel global menos del $1 \%$ de los casos confirmados reciben tratamiento $(11,12)$. En pacientes en fase indeterminada el éxito del tratamiento es difícil de evaluar y en los casos crónicos su efectividad no es evidente, aunque puede retrasar la progresión de la enfermedad $(11,12)$. El tratamiento con ambos medicamentos está contraindicado en mujeres embarazadas, en personas con problemas cardíacos severos y complicaciones hepáticas y renales. El Nifurtimox y el Benznidazol causan efectos adversos cuya frecuencia e intensidad es mayor en personas adultas $(13,14)$; debido a que el benznidazol tiene menos efectos 
adversos y se requiere menor tiempo de tratamiento, se prefiere como el medicamento de primera elección, sin embargo se pueden presentar algunos efectos adversos que pueden interferir en el cumplimiento del tratamiento (14). En relación con el uso del Nifurtimox, algunos estudios han mostrado ser efectivo en pacientes que no toleran el benznidazol (15).

Existen algunos estudios relacionados con el seguimiento de pacientes con EC tratados con benznidazol o nifurtimox, pero pocos abordan la evaluación de la adherencia al medicamento y los factores de riesgo asociados con la adherencia terapéutica $(16,17)$.

De acuerdo con la OMS, la adherencia terapéutica puede definirse como el grado de aceptación de los pacientes a las recomendaciones del médico o de otros proveedores de atención médica, que incluye la toma de los medicamentos, seguir una dieta, hacer cambios de comportamiento y asistir a las citas médicas (1).

La adherencia terapéutica debe compartirse entre el profesional de la salud, el paciente y el sistema de atención en salud. Dicha relación toma importancia cuando existe una comunicación eficaz, una actitud positiva, empatía moralmente neutra, disponibilidad y calidad en el servicio prestado, ya que han demostrado ser determinantes de la adherencia de los pacientes $(18,19)$. Los aspectos psicosociales del paciente involucran el recurso económico, nivel educativo, falta de redes de apoyo social, condiciones de vida inestable, lejanía del centro de tratamiento, costo del transporte, alto costo y accesibilidad de los medicamentos, disfunción familiar, cultura y creencias populares acerca de la enfermedad y el tratamiento, actitud, percepción y las expectativas del mismo (19).

Para evaluar la adherencia terapéutica existen diversos métodos (directos e indirectos) que cuantifican el medicamento o sus metabolitos en muestras biológicas, evaluaciones médicas y consumo de los medicamentos o mediante autoinformes y autoregistros de los pacientes e informes de personas cercanas al paciente (20,21), mediante la aplicación de cuestionarios como el de MoriskyGreen (22).

Los principales factores que se asocian con el abandono al tratamiento terapéutico de la enfermedad de Chagas y el seguimiento clínico son: 1) pacientes que no se consideran enfermos ya que no presentan signos ni síntomas, 2) desconocimiento de la enfermedad, 3) presencia de efectos adversos, 4) falta del medicamento, 5) problemas del personal médico para contactar al paciente, 6) pacientes con falta de compromiso para recibir y tomar el medicamento, y 7) falta de tiempo de los pacientes para recibir el tratamiento.

Hasta donde tenemos información, en México y Morelos no hay estudios relacionados con la evaluación de la adherencia terapéutica en pacientes con enfermedad de Chagas. 
Arce-Vega et al.

El objetivo de este trabajo fue evaluar la adherencia al tratamiento terapéutico de pacientes con diagnóstico confirmado de enfermedad de Chagas en el Estado de Morelos y los factores de riesgo asociados con su cumplimiento.

\section{Material y Métodos}

Estudio de seguimiento de casos realizado en los meses de junio-agosto del 2014 con una muestra por conveniencia de 20 pacientes adscritos a la Jurisdicción Sanitaria \#1 de la Secretaría de Salud del Estado de Morelos, diagnosticados y confirmados con enfermedad de Chagas en 2013 por el Laboratorio Estatal de Salud Pública de Morelos de acuerdo a la Norma Oficial Mexicana NOM-032SSA2-2010. Los criterios de inclusión fueron: que tuvieran un diagnóstico confirmado de enfermedad de Chagas, que estuvieran en tratamiento terapéutico y que aceptaran participar en el estudio después de haber leído la carta de consentimiento informado que fue aprobada por el Comité de Ética en Investigación del INSP. A pesar de que en Morelos hay más casos reconocidos de enfermedad de Chagas, los Servicios de Salud solo autorizaron información de los 20 casos incluidos en este estudio.

Se aplicó un cuestionario estructurado propuesto por Morisky-Green para valorar el cumplimiento de la adherencia (22), y se consideró como cumplidor si respondió de forma correcta $(\mathrm{No} / \mathrm{Si} / \mathrm{No} / \mathrm{No})$ a las 4 preguntas del cuestionario el cual fue llenado por el encuestador; las preguntas fueron: 1) Olvida alguna vez tomar los medicamentos para tratar su enfermedad?, 2) Toma los medicamentos a las horas indicadas ?, 3) Cuando se encuentra bien, deja de tomar los medicamentos ?, y 4) Si alguna vez se siente mal, deja de tomar el medicamento ?. Posteriormente el encuestador aplicó un cuestionario para registrar los factores de riesgo relacionados con el apego terapéutico que incluyó preguntas de escolaridad, acceso, derecho-habiencia, conocimiento de la enfermedad y nivel socio-económico, entre otras.

El seguimiento de los casos se hizo mediante visita domiciliaria o mediante llamada telefónica (23). Se registró la cantidad del medicamento que consumió el paciente en el tiempo indicado y se realizaron entrevistas semi-estructuradas a 8 pacientes para obtener información sobre posibles factores positivos y negativos asociados a la adherencia, también se entrevistó al responsable estatal del programa de la enfermedad de Chagas para tener información de la perspectiva de la enfermedad de Chagas en Morelos.

El análisis estadístico se realizó con estadística analítica, análisis bivariado con cálculo de $t$ de Student para variables continuas, la prueba de Fisher para variables categóricas y un modelo de regresión logística. Se utilizó el programa estadístico Stata 13.0. 
El estudio se realizó con la autorización del Comité de Ética en Investigación del Instituto Nacional de Salud Pública y de la Secretaría de Salud del Estado de Morelos. Los pacientes participantes fueron informados del objetivo del estudio y firmaron una carta de consentimiento informado (Versión aprobada el 24 de marzo del 2014/PTP: 10, Folio Identificador: 057, Comité de Ética en Investigación, Instituto Nacional de Salud Pública).

\section{Resultados}

Se incluyeron 20 pacientes, con edades entre 2 y 67 años (mediana de 32.5 años de edad) (Cuadro 1). El $80 \%$ de los pacientes reportó que fue detectado como positivo a la enfermedad de Chagas en el tamizaje para donación de sangre y confirmados por el Laboratorio Estatal de Salud Pública (LESPMorelos); el 5\% de los casos se detectó por la presencia de signos (chagoma de induración o Signo de Romaña) y fueron atendidos por las unidades médicas de la Secretaría de Salud de Morelos; en este estudio se incluyeron 3 niños con edades de 2, 7 y 13 años de edad (Cuadro 1). El $75 \%$ de los casos fueron detectados por dependencias de la SSM, el $20 \%$ por el IMSS y 5\% por el ISSSTE. El $35 \%$ de los pacientes encuestados confirmaron la presencia de triatóminos en sus hogares y solo 6 (30\%) reportó haber sido picado por lo menos en alguna ocasión, y el 75\% de los casos reportó tener mascotas como perros y gatos. El 90\% de los casos incluidos en este estudio no tenía información sobre la enfermedad de Chagas y el 75\% refirió haber obtenido información del personal de salud. Todos los casos fueron tratados con benznidazol, excepto uno que recibió nifurtimox (Cuadro 1). El $65 \%$ de los casos fueron adherentes según el test de Morisky-Green (22) ya que adoptaron actitudes correctas respecto al tratamiento; el 35\% de los pacientes se consideraron no-adherentes debido a que manifestaron conductas de incumplimiento del tratamiento. Los participantes iniciaron el tratamiento desde una semana hasta 5 meses después de haber sido diagnosticados (Cuadro 1) y la mayoría mencionó haber recibido indicaciones médicas relacionadas con el tratamiento. El $65 \%$ de los pacientes fueron de nivel socio-económico bajo. Del total de la muestra $(n=20)$, el $35 \%$ presentó alguno de los siguientes efectos adversos: náuseas (20\%); exantema (15\%); prurito (10\%), fiebre (10\%); edema facial (5\%); agotamiento (5\%); eritema (5\%); vómito (5\%) y gastritis (5\%). En los dos casos que suspendieron el medicamento se registró náusea, vómito, fiebre, exantema, prurito y edema (Cuadro 1). Respecto a la adherencia, no se observó asociación estadística en las variables de nivel socioeconómico, escolaridad, sexo, accesibilidad al servicio médico y barreras para los estudios clínicos. De los 13 pacientes adherentes, cuatro consideraron tener barreras para los estudios clínicos. Los casos masculinos tuvieron mayor adherencia al tratamiento (75\%) que las mujeres. Al final del 
Arce-Vega et al.

tratamiento estaban pendientes los estudios clínicos y de laboratorio de los pacientes de acuerdo a la normatividad vigente.

\section{Discusión}

Uno de los principales problemas para el tratamiento de pacientes con EC en México y en Morelos, es el acceso, el seguimiento de los casos y la disponibilidad de los medicamentos; generalmente se administra el medicamento existente y no el medicamento de primera elección. De los dos medicamentos (Benznidazol y Nifurtimox) disponibles en México, todas las entidades federativas endémicas utilizan preferentemente el Nifurtimox que el gobierno federal adquiere como donativo a través de la Organización Panamericana de la Salud (OPS), excepto el estado de Morelos que compra el Benznidazol a través de un distribuidor privado, sin embargo, la Secretaría de Salud de Morelos tiene también disponible el Nifurtimox para su uso cuando sea necesario.

Los resultados de este trabajo mostraron congruencia con otros estudios donde midieron adherencia al medicamento y registraron los factores de riesgo para el apego al tratamiento (24). Algunos factores relacionados al tratamiento son los períodos largos, dosis complicadas y efectos adversos, así como la edad del paciente, la escolaridad, las barreras de acceso al servicio de salud y el nivel socioeconómico, entre otros (25). En este estudio, 2 casos suspendieron definitivamente el tratamiento debido a efectos adversos severos y no se ofreció otra alternativa de tratamiento. Los tres niños (2, 7 y 13 años de edad) incluidos en este estudio, era recomendable que fueran tratados con el benznidazol pediátrico $(12.5 \mathrm{mg})$, sin embargo aún no está disponible en México. Ante la falta de otros medicamentos para el tratamiento de la enfermedad de Chagas se requieren estudios que evalúen la efectividad de tratamientos mixtos con medicamentos antiparasitarios como el publicado en 2013 por Perez-Mazliah et al, quienes mostraron en un estudio piloto la reducción de la carga parasitaria en 11 pacientes chagásicos crónicos tratados secuencialmente con alopurinol y benznidazol (29); el tratamiento secuencial fue bien tolerado y hubo una disminución significativa de anticuerpos contra T.cruzi. Del mismo modo un trabajo publicado por Alvarez et al, mostraron que el 20\% (16/81) de pacientes chagásicos que interrumpieron el tratamiento con benznidazol debido a efectos adversos, cumplieron el criterio de cura (30). En el presente estudio 19 de los 20 casos recibieron tratamiento por más de 30 días, por lo que es factible pensar en el efecto curativo del medicamento, sin embargo no existen datos de laboratorio (marcadores biológicos) que apoyen esta posibilidad. Es evidente que se requieren nuevos medicamentos más efectivos, con menos efectos adversos, con menor tiempo de tratamiento, de bajo costo y accesibles a los pacientes en países endémicos (31). En un estudio piloto publicado en 2015, Alvarez et al (32) mostraron que la administración intermitente de benznidazol puede ser un nuevo esquema de tratamiento en pacientes con enfermedad de Chagas. 
Uno de los autores del presente trabajo (CR) participa en la Plataforma de Investigación Clínica de la enfermedad de Chagas que es apoyada por la agencia Drugs for Neglected Diseases Initiative (DNDi), donde anualmente se analizan los avances en el desarrollo de nuevos medicamentos, mejores estrategias de diagnóstico y desarrollo de marcadores biológicos indicadores de curación, entre otros.

En el presente trabajo, el 35\% de los pacientes en estudio presentaron reacciones adversas, dato que coincide con el reportado en otros estudios (26) donde encontraron efectos adversos como neuropatías, reacciones cutáneas graves, síntomas gastrointestinales y/o alteraciones del hemograma, función renal y hepática. Este estudio mostró que existen deficiencias en la prestación del servicio, ya que el período entre el diagnóstico y el tratamiento varió de 3-197 días debido a falta de personal para el seguimiento de los casos o la carencia de los medicamentos, entre otras (Cuadro 1).

En enfermedades que precisan un tratamiento a largo plazo como la enfermedad de Chagas, la disminución de la dosis del medicamente puede tener menos efectos adversos y mejora la adherencia. Un estudio publicado por Pinazo et al, indica que la concentración del benznidazol en el suero no parece estar relacionado con los afectos adversos detectados en 54 pacientes crónicos que fueron sometidos a tratamiento, sin embargo señalan la necesidad de otros estudios para establecer la dosis óptima del medicamento y la reducción del tiempo de tratamiento (27).

De igual manera, se observó que los pacientes en ocasiones no son adherentes al tratamiento debido a que no presentan signos o síntomas y su percepción de salud es óptima; en otros casos, los pacientes prefieren adaptarse a los síntomas que seguir un esquema de tratamiento (28). En este estudio, la visita domiciliaria y/o la llamada telefónica, coadyuvaron al cumplimiento del tratamiento y a la notificación de los efectos adversos. La entrevista directa con los pacientes y la consejería aportada es un factor importante que puede coadyuvar al cumplimiento de la adherencia al tratamiento y al registro de los efectos adversos. El presente estudio reportó que el $65 \%$ de los pacientes fueron adherentes al tratamiento y $35 \%$ presentaron reacciones adversas (leves a moderadas, excepto dos casos con afectos severos) (Cuadro 1), dato que coincide con el reportado por otros autores. El nivel socio-económico bajo y el nivel de escolaridad, pudieron haber influido en el cumplimiento del tratamiento y en el reconocimiento de los efectos adversos; resultados similares han sido reportados por otros autores (24). Otra característica que favorece el apego al tratamiento es el estado civil, en los pacientes casados o que viven con su pareja adoptan actitudes positivas y se forman vínculos de ayuda (25).

Las dimensiones de calidad para mejorar la adherencia terapéutica pueden ser técnica, es decir, que el personal use el conocimiento y técnicas para la solución del problema y/o interpersonal donde el 
Arce-Vega et al.

proveedor del servicio forme un vínculo con el paciente que ayude a crear confianza y estimule el seguimiento correcto de los medicamentos administrados. El sistema de salud puede estructurar acciones prioritarias dirigidas a monitorear el comportamiento de la adherencia terapéutica de los pacientes a través del propio personal de salud. Los médicos y enfermeras pueden asignarse un tiempo en la consulta para revisar la adherencia terapéutica. Los pacientes demuestran mejor adhesión al tratamiento cuando reciben atención del mismo profesional en el transcurso del tiempo. El sistema de comunicación con los pacientes es fundamental, tanto en la institución de salud, en la comunidad, el hogar y la farmacia, entre otros.

En el presente trabajo la entrevista personal con los casos fue importante para ofrecerles información sobre la enfermedad y el tratamiento, incluyendo el registro de los efectos adversos y la atención médica. El responsable de la enfermedad de Chagas en los Servicios de Salud de Morelos estuvo de acuerdo en actualizar al personal médico y operativo, y garantizar la disponibilidad de los medicamentos.

Como resultado de este estudio se recomienda tomar en consideración los siguientes puntos: 1) contar con personal médico que tenga conocimientos básicos de la enfermedad de Chagas y dar consejería a los pacientes y familiares, 2) tener disponibilidad de los medicamentos, 3) disponer de los medicamentos en presentación pediátrica, 4) incorporar los medicamentos en el cuadro básico, 5) proporcionar información clara de la enfermedad de Chagas a los pacientes y familiares, 6) capacitar al personal médico en el registro de caso, 7) facilitar la comunicación entre el Laboratorio Estatal de Salud Pública, las Jurisdicciones Sanitarias, los Centros de Salud y los Hospitales, 8) implementar un manual de atención del paciente, 9) facilitar al paciente el acceso oportuno para las evaluaciones clínicas y de laboratorio, y 10) disminuir el tiempo entre el diagnóstico y el tratamiento.

\section{Agradecimientos}

Los autores agradecemos la valiosa colaboración de todos los pacientes que participaron en este estudio. Al Biól. Marco Ocampo de los Servicios de Salud de Morelos por apoyarnos con la información de los pacientes y a la M. en C. Yury Ana Andrade Zepeda por su apoyo en el análisis de los datos. Nuestro reconocimiento a la C. Aida Ahumada por su invaluable apoyo secretarial. 


\section{Referencias}

1. World Health Organization. Chagas disease in Latin America: an epidemiological update based on 2010 estimates. World Health Organization Weekly Epidemiological Record. 2015 Jun; 90(6): 33-44.

2. Schmunis GA \& Yadon ZE. Chagas disease: a Latin American health problem becoming a world health problem. Acta Trop. 2010 Jul-Aug; 115 (1-2):14-21.

3. Bern C \& Montgomery SP. An estimate of the burden of Chagas disease in the United States. Clin Infect Dis. 2009 Sep; 49 (5): e52-e54.

4. Basile L, Jansá JM, Carlier Y, Salamanca DD, Angheben A, Bartoloni A et al. Chagas disease in European countries: the challenge of a surviellance system. Eurosurveillance. 2011 Sep; 16(37): 19968.

5. Manne-Goehler J, Reich MR, Wirtz VJ. Access to care for Chagas disease in the United States: a health systems analysis. Am J Trop Med Hyg. 2015 May; 93(1): 5.

6. Manne JM, Snively CS, Ramsey JM, Ocampo M, Barnighausen T, Reich MR. Barriers to treatment access for Chagas disease in Mexico. PLOS Neglect Trop Dis. 2013 Oct; 7 (10): e2488.

7. Ramsey JM, Elizondo-Cano M, Sánchez-González G, Peña-Nieves A, Figueroa-Lara A. Opportunity cost for early treatment of Chagas disease in México. PLOS Negl Trop Dis. 2014 Apr; 8(4): e2776.

8. Boletín Epidemiológico, Sistema Nacional de Vigilancia Epidemiológica, Sistema Único de Información, DGE/SSA, http://www.epidemiologia.salud.gob.mx/informes/index.html 2016.

9. Ribeiro I, Sevsick AM, Alves F, Diap G, Don R et al. New, improved treatments for Chagas disease: from the R\&D pipeline to the patients. PLOS Neglect Trop Dis. 2009 Jul; 3 (7):e484.

10. Bern C. Antitrypanosomal therapy for chronic Chagas'disease. N Engl J Med. 2011 Jun 30; 364(26): 2527-34.

11. Viotti R, Alarcon de Noya B, Araujo-Jorge T. Towards a paradigm shift in the treatment of chronic Chagas disease. Animicrob Agents Chemother. 2014; 58: 635-9.

12. Morillo CA, Marin-Neto JA, Avezum A. Randomized trial of benznidazole for chronic Chagas'disease cardiomyopathy. N Engl J Med. 2015 Oct; 373 (1):1295-1306.

13. Jackson Y, Alirol E, Getaz L, Wolff H, Combescure C, Chappuis F. Tolerance and safety of nifurtimox in patients with chronic Chagas disease. Clin Infect Dis. 2010 Nov; 51(10):e69-75.

14. Viotti R, Vigliano C, Lococo B, Alvarez MG, Bertocchi G, Armenti A. Side effects of benznidazole as treatment in chronic Chagas disease: fears and realities. N Engl J Med. 2015 Oct; 373:1295-1306.

15. Perez-Molina JA, Sojo-dorado J, Norman F. Nifurtimox therapy for Chagas disease does not cause hypersensitivity reactions in patients with such previous adverse reactions during benznidazole treatment. Acta Trop. 2013 Aug; 127(2): 101-4.

16. Tornheim JA, Lozano DF, Gilman RH, Castellon M, Solano MA, Sullca W, Torrico F, Bern C. Improved completion rates and characterization of drug reactions with an intensive Chagas disease treatment program in rural Bolivia. PLOS Neglect Trop Dis. 2013 Sep; 7 (9):e2407.

17. DasNeves Pinto A, da Costa V, Rodriguez Coura J, da Silva SA, Verissimo AC, Santos LC, Gomes A $\mathrm{Jr}$, de Macedo RC. Clinical follow-up of responses to treatment with benznidazol in Amazon: A cohort study of acute Chagas disease. PLOS One. 2013 May; 8 (5): e64450.

18. Yurelis GA. La adherencia terapéutica. Rev Cubana Med Gen Integr. 2001; 17(5): 502-505.

19. Renato Z, John DM, Jorge PV, José FA. Percepción de la adherencia a tratamientos en pacientes con factores de riesgo cardiovascular. Rev Fac Nac Salud Pública [online], 2012 Mayo-Agosto; 30(2), 163-174.

20. Cramer Jam Manson RH, Prevey ML, Scheyer RD, Ouellette VL. How often is medication taken as prescribed?. A novel assessment technique. JAMA. 1989; 261(22):3273-3277.

21. Larrea $\mathrm{P}, \mathrm{Mir} \mathrm{M}$. Adherencia al tratamiento en el paciente anciano. Información terapéutica del Sistema Nacional de Salud 2004; 28(5): 1453-5. http://www.msc.es/farmacia/infmedic

22. Morisky DE, Green LW, Levine DM. Concurrent and predictive validity of a self-reported measure 
Arce-Vega et al.

of medication adherence. Med Care. 1986 Jan; 24(1):67-74.

23. Peralta ML \& Carbajal P. Adherencia al tratamiento. Rev Cent Dermatol Pascua 2008 Sep-Dic; 17(3): 84-88.

24. Tornheim JA, Lozano DF, Gilman RH, Castellon M, Solano MA, Sullca W, Torrico F, Bern C. Improved completion rates and characterization of drug reactions with an intensive Chagas disease treatment program in rural Bolivia. PLOS Negl Trop Dis. 2013 Sept; 7 (9); e2407.

25. Arrivillaga-Quintero M. Análisis de las barreras para la adherencia terapéutica en mujeres Colombianas con VIH/SIDA: cuestión de derechos de salud. Salud Pública Mex. 2010 Jul-Aug; 52(4):350-6.

26. Pinazo MJ, Muñoz J, Posada E, López-Chejade P, Gallego M. Tolerance of benznidazole in treatment of Chagas'disease in adults. Antimicrob Agents Chemother. 2010 Nov; 54(11): 4896-99

27. Pinazo MJ, Guerrero L, Posada E, Rodriguez E, soy D, Gascon J. Benznidazole-related adverse drug reactions and their relationship to serum drug concentrations in patients with chronic Chagas disease. Antimicrob Agents Chemother. 2013 Jan; 57 (1):390-5.

28. Apt B, Werner. Guías clínicas de la enfermedad de Chagas: Parte I. Introducción y epidemiología. Rev. Chil. infectol., Jun 2008; 25(3):189-193.

29. Perez-Mazliah DE, Alvarez MG, Cooley G, Lococo BE, Bertocchi G, Petti M, Albareda MC, Armenti AH, Tarleton RL, Laucella SA, Viotti R. Sequential combined treatment with allopurinol and benznidazole in the chronic phase of Trypanosoma cruzi infection: a pilot study. J Antimicrob Chemother. 2013 Feb; 68 (2): 424-37.

30. Alvarez MG, Vigliano C, Lococo B, Petti M, Bertocchi G, Viotti R. Seronegative conversion after incomplete benznidazole treatment in chronic Chagas disease. Trans R Soc Trop Med Hyg. 2012 Oct; 106 (10): 636-8.

31. Bermudez J, Davies C, Simonazzi A, Real JP, Palma S. Current drug therapy and pharmaceutical challenges for Chagas disease. Acta Tropica. 2016 Apr; 156: 1-16.

32. Alvarez MG, Hernández Y, Bertocchi G, Fernández M, Lococo B, Ramirez JC, Cura C, Albizu Cl, Schijman A, Abril M, Sosa-Estani S, Viotti R. New scheme of intermittent benznidazole administration in patients chronically infected with Trypanosoma cruzi: a pilot short-term follow-up study with adult patients. Animicrob Agents Chemother. 2015 Oct; 60(2): 833-7. 
Tabla

Información general de los pacientes con diagnóstico de enfermedad de Chagas en tratamiento terapéutico

\begin{tabular}{|c|c|c|c|c|c|c|c|c|c|c|c|c|}
\hline ID & Sexo & Edad & Lugar de origen & $\begin{array}{c}\text { Fecha de } \\
\text { diagnóstico }\end{array}$ & $\begin{array}{c}\text { Inicio de } \\
\text { tratamiento }\end{array}$ & $\begin{array}{l}\text { Dias } \\
\text { Dx-Tx* }\end{array}$ & $\begin{array}{c}\text { Termino de } \\
\text { tratatamiento }\end{array}$ & $\begin{array}{c}\text { Días de suspensión } \\
\text { del tratamientot }\end{array}$ & $\begin{array}{c}\text { Duración } \\
\text { Tx श }\end{array}$ & Efectos adversos & Adherencia & Tratamiento \\
\hline 1001 & Masculino & 7 & Xochitepec & $25 / 10 / 2013$ & $11 / 12 / 2013$ & 47 & $11 / 03 / 2014$ & 6 & 91 & & No & Benznidazol \\
\hline 1003 & Masculino & 38 & Atlacholoaya & $23 / 08 / 2013$ & $16 / 12 / 2013$ & 115 & $28 / 02 / 2014$ & 0 & 75 & Agotamiento & $\mathrm{Si}$ & Benznidazol \\
\hline 1004 & Masculino & 33 & Miguel hidalgo & $12 / 12 / 2013$ & $28 / 01 / 2014$ & 47 & $28 / 03 / 2014$ & 0 & 60 & & $\mathrm{Si}$ & Benznidazol \\
\hline 1005 & Masculino & 13 & Cuernavaca & 09/01/2014 & $01 / 06 / 2014$ & 142 & $31 / 07 / 2014$ & 0 & 61 & & $\mathrm{Si}$ & Nifurtimox \\
\hline 1006 & Masculino & 2 & Cuernavaca & $24 / 07 / 2013$ & $27 / 07 / 2013$ & 3 & $23 / 10 / 2013$ & 26 & 89 & & No & Benznidazol \\
\hline 1007 & Femenino & 28 & Tezoyuca & $11 / 12 / 2013$ & $06 / 04 / 2014$ & 115 & $11 / 04 / 2014$ & Suspensión § & 8 & $\begin{array}{c}\text { Náuseas, vómito, } \\
\text { fiebre, exantema, } \\
\text { prurito, edema }\end{array}$ & No & Benznidazol \\
\hline 1008 & Masculino & 40 & Emiliano zapata & $19 / 08 / 2013$ & $25 / 11 / 2013$ & 98 & $24 / 01 / 2014$ & 0 & 61 & & $\mathrm{Si}$ & Benznidazol \\
\hline 1009 & Femenino & 21 & Jiutepec & $19 / 08 / 2013$ & $04 / 03 / 2014$ & 197 & $09 / 05 / 2014$ & 0 & 67 & Nauseas & $\mathrm{Si}$ & Benznidazol \\
\hline 1010 & Masculino & 62 & Jiutepec & $30 / 10 / 2013$ & $10 / 12 / 2013$ & 41 & $06 / 02 / 2014$ & 0 & 59 & Náuseas, gastritis & $\mathrm{Si}$ & Benznidazol \\
\hline 1012 & Masculino & 56 & Cuernavaca & $07 / 10 / 2013$ & $05 / 03 / 2014$ & 149 & $02 / 05 / 2014$ & 0 & 59 & & $\mathrm{Si}$ & Benznidazol \\
\hline 1013 & Masculino & 43 & Cuernavaca & $07 / 08 / 2013$ & $17 / 12 / 2013$ & 132 & $01 / 03 / 2014$ & 0 & 74 & & $\mathrm{Si}$ & Benznidazol \\
\hline 1014 & Femenino & 27 & Cuernavaca & $13 / 08 / 2013$ & $21 / 08 / 2013$ & 8 & $13 / 10 / 2013$ & 8 & 54 & & No & Benznidazol \\
\hline 1015 & Masculino & 48 & Coatlan del Rio & $19 / 08 / 2013$ & $12 / 12 / 2013$ & 115 & 09/02/2014 & 0 & 60 & & $\mathrm{Si}$ & Benznidazol \\
\hline 1016 & Masculino & 57 & Jiutepec & $26 / 07 / 2013$ & $23 / 08 / 2013$ & 33 & $22 / 09 / 2013$ & Suspensión § & 31 & $\begin{array}{c}\text { Fiebre, eritema, } \\
\text { exantema }\end{array}$ & No & Benznidazol \\
\hline 1017 & Masculino & 32 & Cuernavaca & $19 / 08 / 2013$ & $27 / 01 / 2014$ & 162 & $27 / 03 / 2014$ & 0 & 60 & & $\mathrm{Si}$ & Benznidazol \\
\hline 1018 & Masculino & 23 & Cuernavaca & $07 / 08 / 2013$ & $08 / 11 / 2013$ & 93 & $07 / 01 / 2014$ & 0 & 61 & Náuseas & $\mathrm{Si}$ & Benznidazol \\
\hline 1019 & Masculino & 67 & Temixco & $07 / 10 / 2013$ & $16 / 01 / 2014$ & 101 & $15 / 02 / 2014$ & 0 & 31 & Exantema, prurito & No & Benznidazol \\
\hline 1020 & Masculino & 53 & Jiutepec & $24 / 07 / 2013$ & $27 / 01 / 2014$ & 187 & $27 / 03 / 2014$ & 0 & 60 & & $\mathrm{Si}$ & Benznidazol \\
\hline
\end{tabular}

* Días transcurridos desde el diagnóstico de la enfermedad hasta la primera toma del tratamiento; † Días que se suspendió el tratamiento;

‡Días que transcurrieron desde el inicio del tratamiento hasta su finalización; § Suspensión definitiva; || Diagnóstico confirmado por laboratorio;

If Dias de duración del tratamiento. 